\title{
Multiple bone brown tumor secondary to primary hyperparathyroidism: a case report and literature review
}

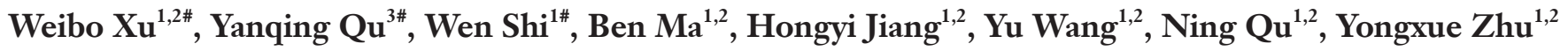 \\ ${ }^{1}$ Department of Head and Neck Surgery, Fudan University Shanghai Cancer Center, Shanghai 200032, China; ${ }^{2}$ Department of Oncology, Shanghai \\ Medical College, Fudan University, Shanghai 200032, China; ${ }^{3}$ Department of Thyroid Surgery, Yantaishan Hospital, Yantai 264001, China \\ \#These authors contributed equally to this work. \\ Correspondence to: Yongxue Zhu; Ning Qu; Yu Wang. Department of Head \& Neck Surgery, Fudan University Shanghai Cancer Center, No. 270, \\ Dong’an Road, Shanghai 200032, China. Email: zhuyongxuelb@icloud.com; jonathan_qn@163.com; neck130@sina.com.
}

\begin{abstract}
Bone brown tumors secondary to primary hyperparathyroidism (PHPT) are rare and only around $2-5 \%$ of PHPT patients have multiple bone brown tumor lesions, which are also uncommon in literatures. We found a female patient who got misdiagnosis of multiple malignant bone tumors in our clinical work, she was eventually diagnosed as a brown tumor secondary to hyperparathyroidism. This article records the diagnosis and treatment process, and summarizes similar case reports in the past decade to provide experience in diagnosis and treatment of similar cases that may occur in the future.
\end{abstract}

Keywords: Bone brown tumor; primary hyperparathyroidism (PHPT); diagnosis and treatment; case report; literature review

Submitted Jul 16, 2019. Accepted for publication Sep 27, 2019.

doi: $10.21037 /$ gs.2019.11.14

View this article at: http://dx.doi.org/10.21037/gs.2019.11.14

\section{Introduction}

Hyperparathyroidism is mainly divided into three types: primary, secondary and tertiary hyperparathyroidism, primary hyperparathyroidism (PHPT) is a phenomenon of excessive secretion of parathyroid hormone (PTH) caused by excessive hyperplasia, neoplasia, and carcinogenesis of parathyroid. The disease is commonly occurred in white race, the worldwide incidence rate of people under 50 years old is about $20 / 100,000$, and the incidence rate is lower in yellow people (1).

It has been reported that $2-5 \%$ of patients with PHPT have brown tumor (osteitis fibrosa cystica) (2,3). Bone brown tumor secondary to PHPT is very rare, which is caused by massive secretion of PTH. First, a large amount of bone calcium is mobilized into blood, resulting in fibrous tissue hyperplasia in bone and leaky hemorrhage. Then macrophage reactive hyperplasia resulted in phagocytosis of red blood cells and a large amount of hemosiderin is produced, thereby forming a brown nodule. The lesions are usually multiple, most commonly seen in ribs, clavicle, pelvis, long bones and mandibles while others are rare (4). The image of brown tumor is often characterized by osteoporosis, thinning of cortical bone and multiple localized cystic bone destruction. Here we report a case of multiple bone brown tumors caused by PHPT in order to provide some reference for future clinical practice.

\section{Case presentation}

A 30-year-old female was admitted to Shanghai Tenth People's Hospital with the chief complaint of pain in the lower back, right lower limb, and left shoulder, and this kind of pain could not be alleviated by taking a rest. SPECT-CT suggested a high probability of bone-marrowderived malignancies and hydronephrosis. Then she went to bone and soft tissue surgery of Fudan University Shanghai Cancer Center for further diagnosis and treatment. 
Enhanced computed tomography (CT) showed there was abnormal bone density in the skull, mandible, part of the vertebral body and scapula on both sides. Meanwhile, a $3 \mathrm{~cm}$-sized nodule at posterior margin of the left thyroid lobe was also detected (Figure 1). Emission CT (ECT) with $99 \mathrm{mTc}-\mathrm{MIBI}$ revealed that posterior left thyroid lobe had an increased intake in both early and delayed stages.

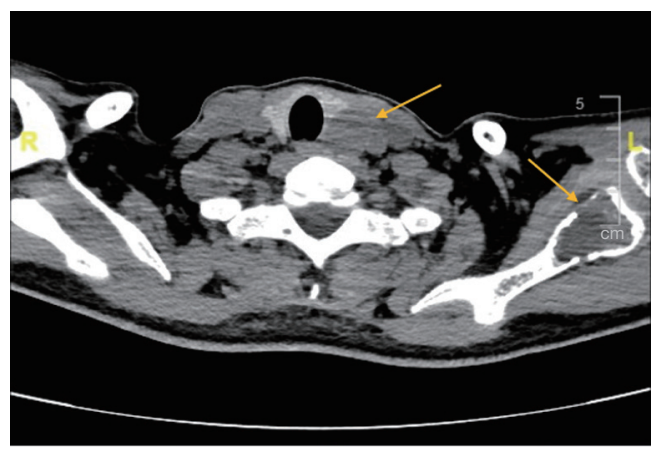

Figure 1 CT suggests enlargement of left parathyroid and bone destruction. Two yellow arrows indicate abnormal bone density in the scapula and a $3 \mathrm{~cm}$-sized nodule at posterior margin of the left thyroid lobe.
Besides, according to the ECT image we could also find bone destruction in T1 vertebral body, both ends of the left clavicle, bilateral scapula, bilateral ribs, suggesting multi bone metastases (Figure 2).

For definite diagnosis, a biopsy of anterior superior of left iliac spine was performed. Postoperative histological pathology was giant cell lesions, suggesting brown tumor. Considering that the patient had a history of parathyroid nodule for three years and ultrasound displayed multiple scattered echoes in bilateral peripheral of renal pelvis, we tested her blood electrolyte and PTH level. Her PTH was $180.500 \mathrm{pmol} / \mathrm{L}$, much higher than normal level (1.575$6.825 \mathrm{pmol} / \mathrm{L})$; serum calcium level was $2.87 \mathrm{mmol} / \mathrm{L}$ (normal, 2.11-2.52 mmol/L), phosphorus was low at $0.37 \mathrm{mmol} / \mathrm{L}$ (normal, $0.85-1.51 \mathrm{mmol} / \mathrm{L}$ ). In addition to this, ultrasound also suggested a nodule of $37 \times 18 \times 21 \mathrm{~mm}^{3}$ size in posterior left thyroid lobe, which was further confirmed what the ECT image.

Then the patient underwent a parathyroid adenoma resection surgery, the postoperative pathology confirmed it was a $3 \times 2.2 \times 1.2 \mathrm{~cm}^{3}$ parathyroid adenoma (Figures 3,4$)$. One hour after surgery, her PTH was at $13.760 \mathrm{pmol} / \mathrm{L}$. For 24
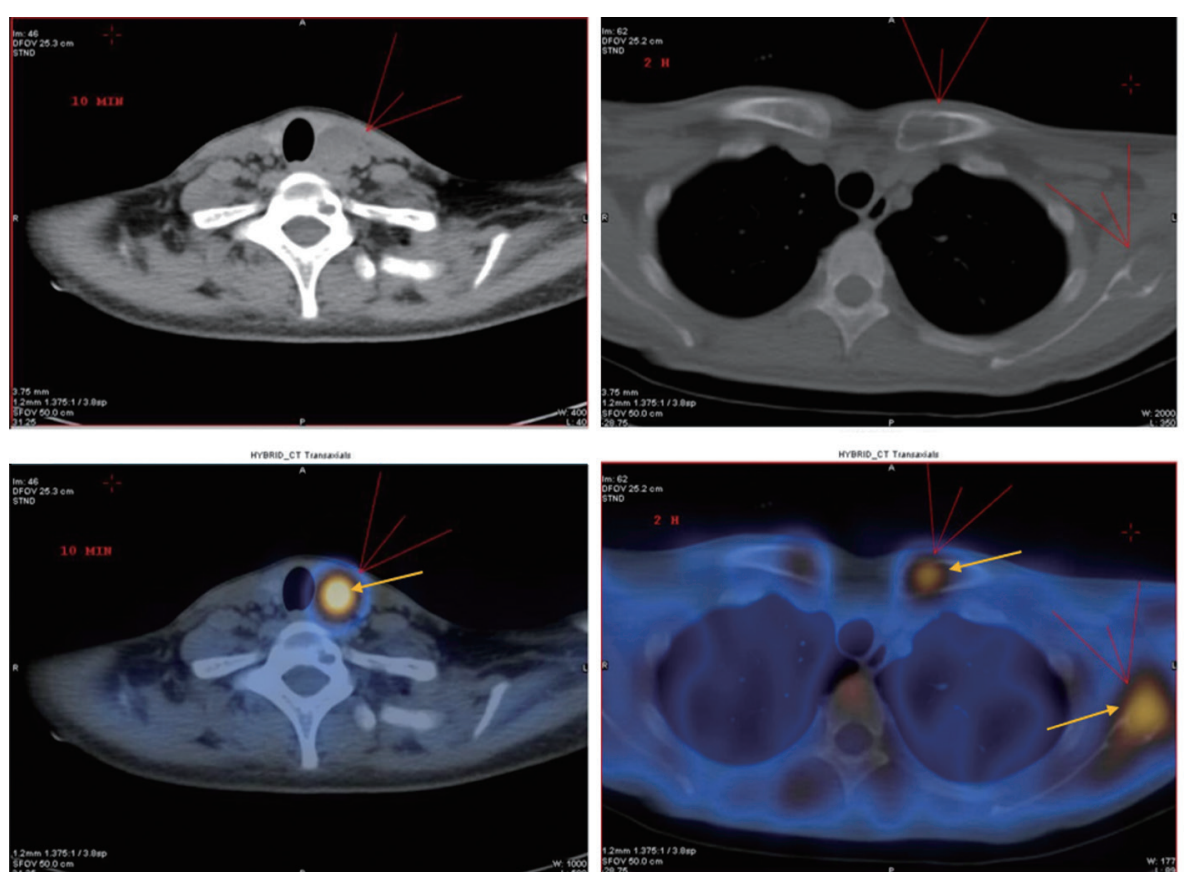
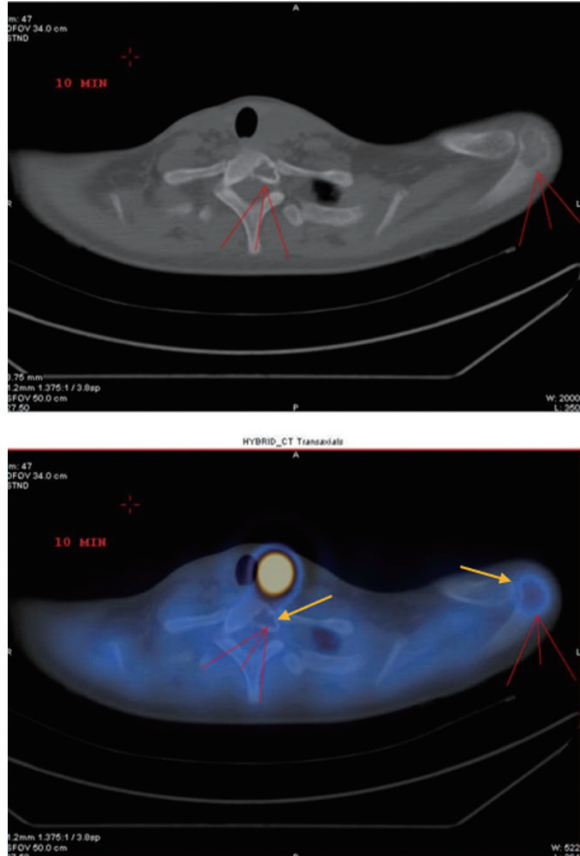

Figure 2 99mTc-MIBI suggests hyperparathyroidism and multiple bone destruction. Yellow arrows indicate bone destruction in T1 vertebral body, both ends of the left clavicle, bilateral scapula and bilateral ribs. 

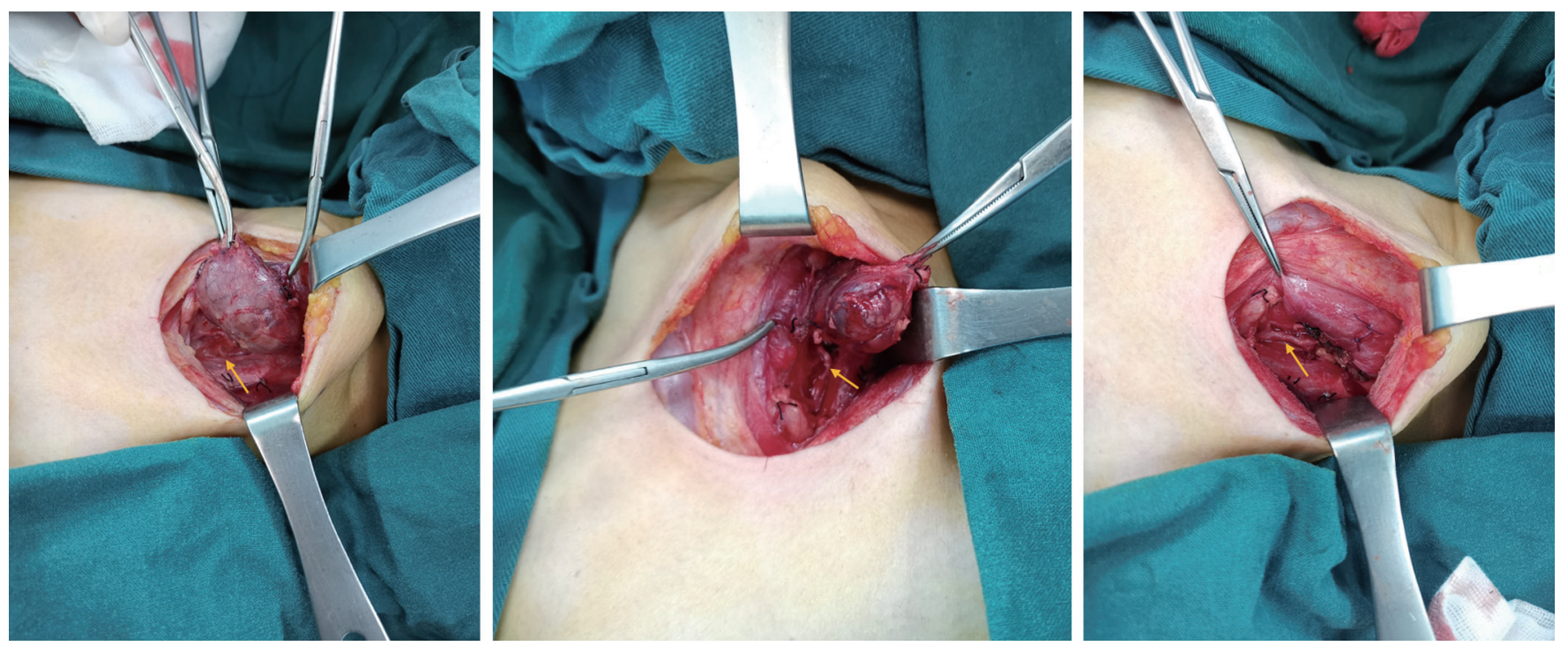

Figure 3 Parathyroid adenoma seen during surgery (showing recurrent laryngeal nerve). Yellow arrows indicate left recurrent laryngeal nerve.
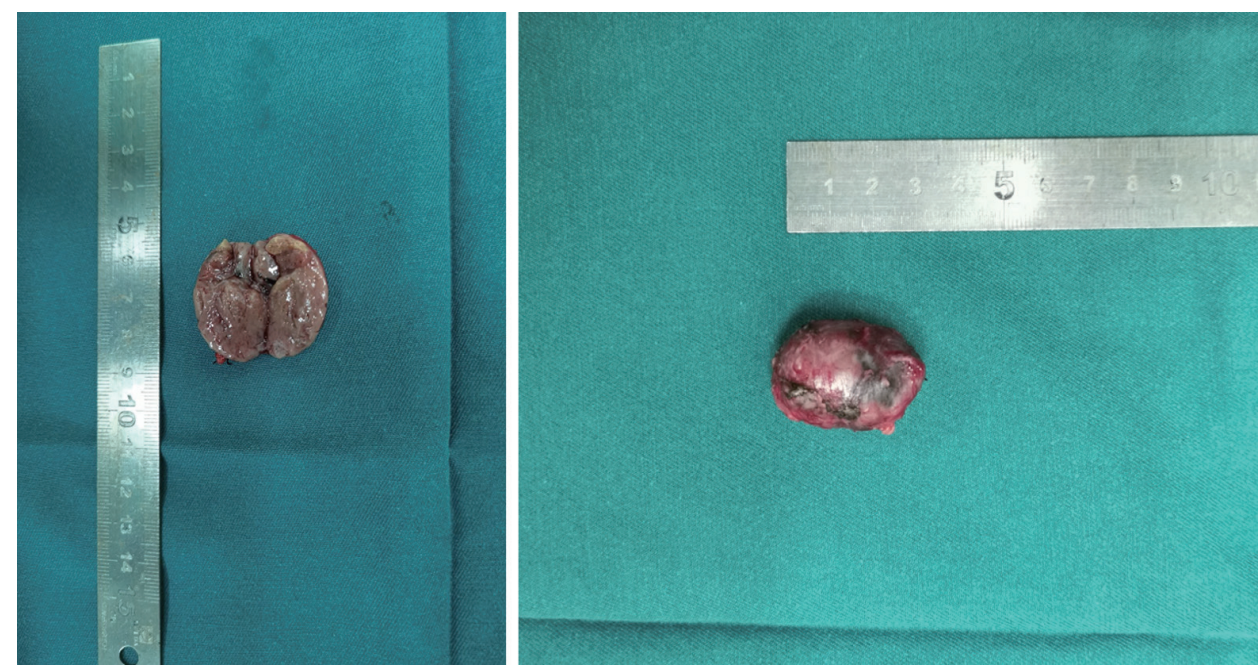

Figure 4 Size of the parathyroid adenoma removed by surgery.

and 48 hours after surgery it was at 5.520 and $0.808 \mathrm{mmol} / \mathrm{L}$, respectively.

At present, this patient has been followed up for three months. PTH and blood calcium level are normal. The imaging manifestation shows no progress in the original brown tumor lesions. The patient has returned life to normal.

\section{Discussion}

Bone brown tumor, also known as osteitis fibrosa cystica, has three stages of progression (5). First of all, elevated PTH secretion stimulates osteoclasts to absorb bone while collagen fibers form in bone marrow. In the second phase, known as the stage of fibrotic osteitis, trabecular bone is absorbed and bone marrow is replaced by loosened fibrosis, hemosiderin-containing macrophages, microfracture hemorrhage area and reactive woven bone. 
As hyperparathyroidism and hemorrhage goes on, cystic degeneration eventually occurs, leading to the final stage of disease (fibrous cystic osteitis). Cysts are the result of intra-bone hemorrhage and tissue degeneration. They are filled with a large number of osteoclasts, macrophages and fibroblasts that are engulfed with hemosiderin. Hemorrhage, hemosiderin and excessive blood vessels lead to brown appearance, so it's called brown tumor. Brown tumors may not show any characteristic changes on either CT or MRI. They may only appear as solid, mixed solid and cystic lesions, or cystic lesions (6).

Because it is uncommon in clinical practice, and its clinical symptoms and imaging finding are easily confused with malignant bone tumors, brown tumor is difficult to diagnose, leading to delayed treatment and economic losses for patients. In this case, the patient had obvious symptom of multiple bone pain. The first hospital made a diagnosis of multiple metastases of malignant bone tumor after performing SPECT-CT, and suggested her to consult to bone and soft tissue surgery of our hospital for exhaustive examination. Nuclear medicine examination in our hospital also tended to the same diagnosis. However, after biopsy and comprehensive inquiry of medical history, we found this patient had symptoms associated with hyperparathyroidism and urinary calculi. Cervical ultrasound results further confirmed the enlargement of left parathyroid. Therefore, this patient was eventually diagnosed with brown tumor secondary to PHPT and underwent surgical resection of the parathyroid.

To further explore the characteristics of bone brown tumor secondary to PHPT, concerning blood tests feature, treatments, and prognosis, we collected similar cases reported in the past decade. Entry criteria are as follows: searching in the PubMed database (https://www. ncbi.nlm.nih.gov/pubmed/) with the keyword "(brown tumor)" AND (Primary Hyperparathyroidism) and published articles during 2009-2019 recorded in the SCI were included, meta-analysis and non-English writing articles were excluded. Finally, data of age, sex, blood test results (multiple of the highest or lowest reference value), treatment methods, with or without urinary calculi, causes of PHPT, size of parathyroid, and prognosis were analyzed in 31 cases of 24 articles (7-30). These characters of all cases are shown in Table 1. The average age of 31 patients was $41.55 \pm 15.50$ years old, including 11 males $(35.5 \%)$ and 20 female patients $(64.5 \%)$. Serum calcium, PTH and ALP were significantly elevated in most patients.
Serum phosphorus was correspondingly reduced. Urinary calculi occurred in $4(12.9 \%)$ of all patients. All patients underwent parathyroidectomy. There were 2 cases $(6.5 \%)$ got postoperative pathological diagnosis of parathyroid carcinoma and 1 case $(3.2 \%)$ was simple parathyroid hyperplasia, and the remaining 28 cases $(90.3 \%)$ were parathyroid adenoma. Except for 4 patients who had no reported prognosis, only 1 patient with parathyroid carcinoma had recurrence and underwent secondary surgery in the remaining 27 patients. Others did not recur during the follow-up period, and the brown tumor lesions also appeared to shrink, which basically returned normal life.

This type of case is seldom seen in epidemiology. The patient disease may be delayed for a long time due to unclear diagnosis, as a result, it is easy to form a large parathyroid mass. Through reflection of whole process of diagnosis and treatment of this case and literature review, for patients with multiple bone pain, screening of cervical ultrasound, PTH, serum calcium and phosphorus should be performed to exclude multiple bone destruction caused by PHPT. Now that PTH has a short half-life time ranging from several minutes to dozens of minutes in blood (31), clear diagnosis and surgical resection of parathyroid as early as possible can yield good therapeutic results. As for brown tumor lesions, generally, there is no need for surgical treatment, but a small number of patients with large lesions still need surgical resection and implanting bone cement to restore function (32).

\section{Acknowledgments}

None.

\section{Footnote}

Conflicts of Interest: The authors have no conflicts of interest to declare.

Ethical Statement: The authors are accountable for all aspects of the work in ensuring that questions related to the accuracy or integrity of any part of the work are appropriately investigated and resolved. Written informed consent was obtained from the patient according to institutional guidelines for publication of this case report and any accompanying images.

\section{References}




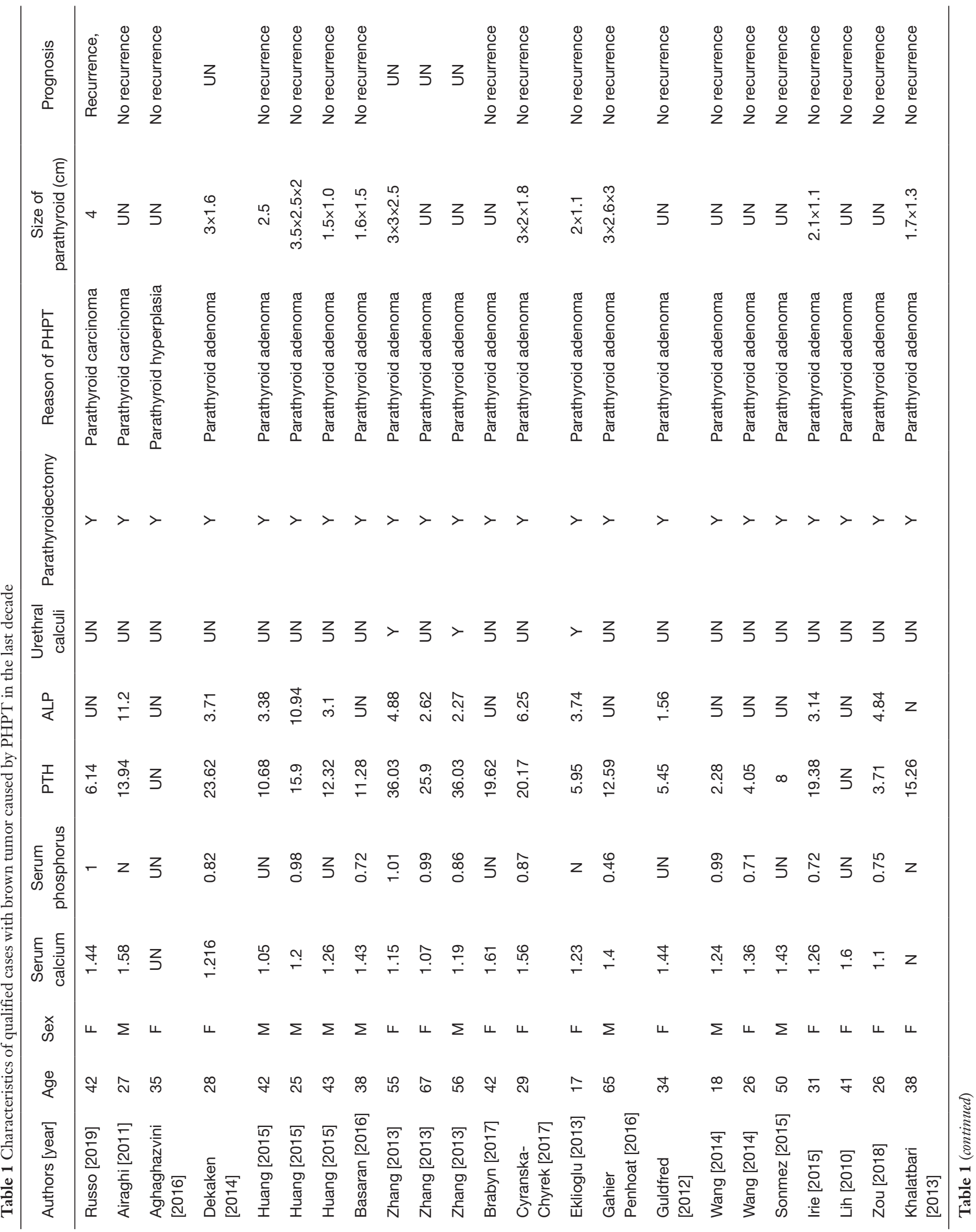




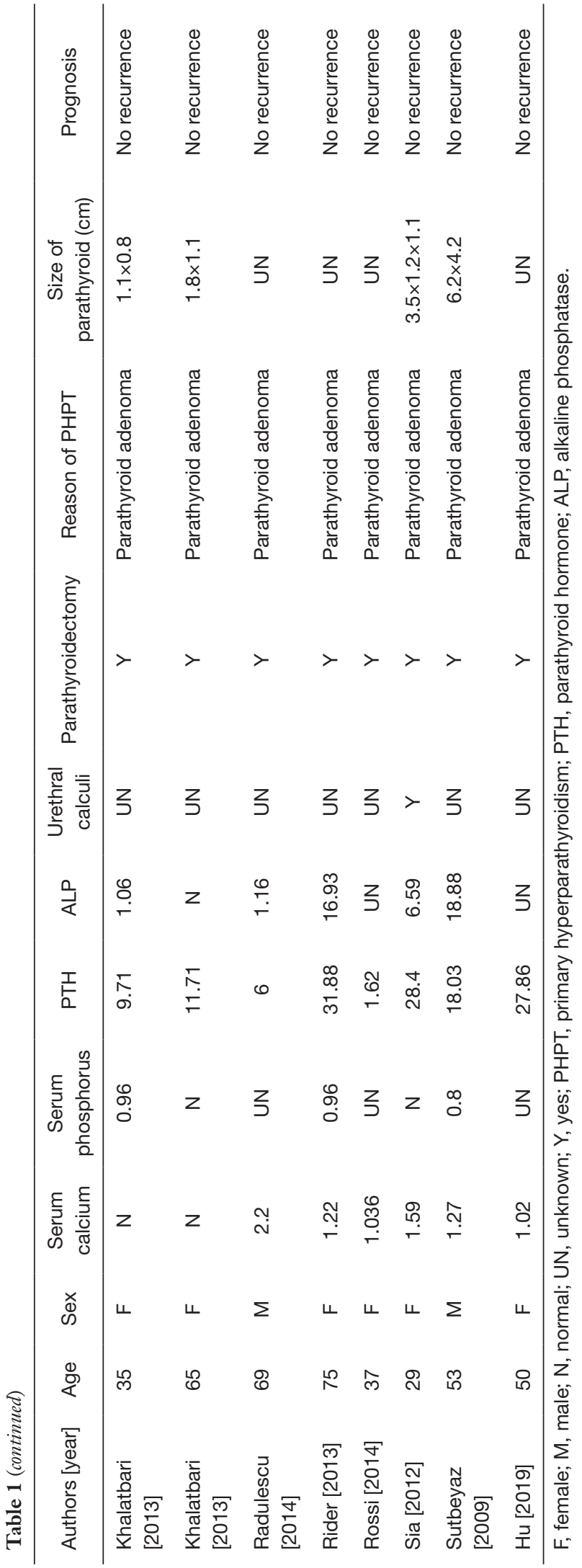

1. Yeh MW, Ituarte PH, Zhou HC, et al. Incidence and prevalence of primary hyperparathyroidism in a racially mixed population. J Clin Endocrinol Metab 2013;98:1122-9.

2. Silverberg SJ, Bilezikian JP. Evaluation and management of primary hyperparathyroidism. J Clin Endocrinol Metab 1996;81:2036-40.

3. Keyser JS, Postma GN. Brown tumor of the mandible. Am J Otolaryngol 1996;17:407-10.

4. Azria A, Beaudreuil J, Juquel JP, et al. Brown tumor of the spine revealing secondary hyperparathyroidism. Report of a case. Joint Bone Spine 2000;67:230-3.

5. Subramanian S, Viswanathan VK. Lytic Bone Lesions. StatPearls. Treasure Island (FL), 2019.

6. Hong WS, Sung MS, Chun KA, et al. Emphasis on the MR imaging findings of brown tumor: a report of five cases. Skeletal Radiol 2011;40:205-13.

7. Aghaghazvini L, Sharifian H, Rasuli B. Primary Hyperparathyroidism Misdiagnosed as Giant Cell Bone Tumor of Maxillary Sinus: A Case Report. Iran J Radiol 2016;13:e13260.

8. Airaghi L, Pisano G, Pulixi E, et al. Unusual presentation in a case of primary hyperparathyroidism. J Res Med Sci 2011;16:1078-81.

9. Basaran Y, Ince S, Alagoz E, et al. An unusual presentation of primary hyperparathyroidism: multiple brown tumors and coexisting thyroid carcinoma. Rev Esp Med Nucl Imagen Mol 2016;35:321-4.

10. Huang R, Zhuang R, Liu Y, et al. Unusual presentation of primary hyperparathyroidism: report of three cases. BMC Med Imaging 2015;15:23.

11. Dekaken A, Gouri A, Bentorki AA, et al. Knee brown tumor revealing a primary hyperparathyroidism: a case report. Ann Biol Clin (Paris) 2014;72:245-8.

12. Russo M, Borzi G, Ilenia $M$, et al. Challenges in the treatment of parathyroid carcinoma: a case report. Hormones (Athens) 2019;18:325-8.

13. Zhang J, Wang H, Tian W, et al. Brown tumor of the rib as a first presentation of primary hyperparathyroidism: Report of three cases and literature review. Thorac Cancer 2013;4:474-8.

14. Eklioglu BS, Atabek ME, Akyurek N. Parathyroid adenoma presented with multiple brown tumors and nephrocalcinosis. J Pediatr Endocrinol Metab 2013;26:213-4.

15. Cyranska-Chyrek E, Szczepanek-Parulska E, Markuszewski J, et al. Sudden Hip Pain in a Young 
Woman. Am J Med 2017;130:e379-81.

16. Wang $X$, Wang $M$, Zhang J, et al. Humeral brown tumor as first presentation of primary hyperparathyroidism caused by ectopic parathyroid adenomas: report of two cases and review of literature. Int J Clin Exp Pathol 2014;7:7094-9.

17. Guldfred LA, Daugaard S, von Buchwald C. Brown tumor mimicking maxillary sinus mucocele as the first manifestation of primary hyperparathyroidism. Auris Nasus Larynx 2012;39:418-21.

18. Irie $T$, Mawatari $T$, Ikemura $S$, et al. Brown tumor of the patella caused by primary hyperparathyroidism: a case report. Korean J Radiol 2015;16:613-6.

19. Sonmez E, Tezcaner T, Coven I, et al. Brown Tumor of the Thoracic Spine: First Manifestation of Primary Hyperparathyroidism. J Korean Neurosurg Soc 2015;58:389-92.

20. Lih A, Lewis M, Carter J. A rare case of primary hyperparathyroidism and osteitis fibrosa cystica. Med J Aust 2010;193:416.

21. Zou H, Song L, Jia M, et al. Brown tumor of multiple facial bones associated with primary hyperparathyroidism: A clinical case report. Medicine (Baltimore) 2018;97:e11877.

22. Radulescu D, Chis B, Donca V, et al. Brown tumors of the femur and pelvis secondary to a parathyroid carcinoma: report of one case. Rev Med Chil 2014;142:919-23.

23. Rider T, Baig I, Sayer C, et al. Pathological finger fracture. BMJ 2013;346:f1024.

24. Khalatbari MR, Hamidi M, Moharamzad Y, et al. Brown tumors of the anterior skull base as the initial manifestation of true normocalcemic primary hyperparathyroidism:

Cite this article as: $\mathrm{Xu} W, \mathrm{Qu} \mathrm{Y}$, Shi W, Ma B, Jiang H, Wang Y, Qu N, Zhu Y. Multiple bone brown tumor secondary to primary hyperparathyroidism: a case report and literature review. Gland Surg 2019;8(6):810-816. doi: 10.21037/ gs.2019.11.14 report of three cases and review of the literature. Turk Neurosurg 2013;23:260-6.

25. Rossi B, Ferraresi V, Appetecchia ML, et al. Giant cell tumor of bone in a patient with diagnosis of primary hyperparathyroidism: a challenge in differential diagnosis with brown tumor. Skeletal Radiol 2014;43:693-7.

26. Sia HK, Hsieh MC, Yang LH, et al. Maxillary brown tumor as initial presentation of parathyroid adenoma: a case report. Kaohsiung J Med Sci 2012;28:400-3.

27. Sutbeyaz Y, Yoruk O, Bilen H, et al. Primary hyperparathyroidism presenting as a palatal and mandibular brown tumor. J Craniofac Surg 2009;20:2101-4.

28. Hu J, He S, Yang J, et al. Management of brown tumor of spine with primary hyperparathyroidism: A case report and literature review. Medicine (Baltimore) 2019;98:e15007.

29. Brabyn P, Capote A, Belloti M, et al. Hyperparathyroidism Diagnosed Due to Brown Tumors of the Jaw: A Case Report and Literature Review. J Oral Maxillofac Surg 2017;75:2162-9.

30. Gahier Penhoat M, Drui D, Ansquer C, et al. Contribution of 18-FDG PET/CT to brown tumor detection in a patient with primary hyperparathyroidism. Joint Bone Spine 2017;84:209-12.

31. Nguyen-Yamamoto L, Rousseau L, Brossard JH, et al. Origin of parathyroid hormone (PTH) fragments detected by intact-PTH assays. Eur J Endocrinol 2002;147:123-31.

32. Guney E, Yigitbasi OG, Bayram F, et al. Brown tumor of the maxilla associated with primary hyperparathyroidism. Auris Nasus Larynx 2001;28:369-72. 